\title{
The State of Health Information Exchange
}

\author{
Joshua M. Liao ${ }^{1}$ and Danny Chu ${ }^{1,2,3 *}$
}

${ }^{1}$ Division of Cardiothoracic Surgery, Michael E. DeBakey Department of Surgery, Baylor College of Medicine, Houston, TX, USA

${ }^{2}$ Michael E. DeBakey VA Medical Center, Houston, TX, USA

${ }^{3}$ Division of Cardiovascular Surgery, the Texas Heart Institute at St. Luke's Episcopal Hospital, Houston, TX, USA

Keywords: Electronic health records; Health information exchange; Information systems; Medical records; Regional health information organization

Abbreviations: Electronic Health Records (EHR); Health Information Exchange (HIE); Regional Health Information Organization (RHIO)

\section{Introduction}

Since 1998, when the US government committed approximately 30 billion dollars over 10 years [1] to promoting the use of electronic health records (EHRs) through the Health Information Technology for Economic and Clinical Health Act, a great deal of effort has been made to address how EHRs could be adopted by US care providers and used to improve quality of care through the sharing of important health information. Along with funding, specific meaningful use objectives have been developed for staged implementations [1].

One proposed mechanism for exchanging health information is health information exchange (HIE), defined as the "electronic movement of health-related information among organizations, according to nationally recognized standards" [2]. An analysis of HIE has associated it with meaningful cost savings [3]. An important problem, however, is that many proprietary EHRs are not designed for exchange, and individual institutional attempts to facilitate exchange have proven tedious and expensive. Moreover, existing Web-based mechanisms are still too underdeveloped and technically involved for widespread use [4]. In response, regional health information organizations (RHIOs) have been created to facilitate regional information exchange. These organizations have a simple design intended to allow direct information exchange and rapport among local stakeholders, and they have been received favorably in early pilot testing [5].

While the RHIO is excellent in concept, its sustained implementation may prove challenging. Because they are created within open systems, RHIOs face larger barriers to HIE than do efforts within closed, umbrella systems like that used in the Veterans Health Administration. Also, RHIOs have a considerable failure rate [6].

To better understand the effectiveness of RHIOs around the country and to determine whether they are meeting standards of meaningful use, Adler-Milstein et al. [4] recently reviewed the current incentive structure around HIEs and found that the majority of existing RHIOs did not meet financial viability criteria. Those that did represented a minority of US hospitals and ambulatory practices, and only a small proportion of these hospitals were operational and meeting meaningful use standards that could achieve projected HIE benefits. Although the study was limited by self-reporting and the potential for incomplete capture of all HIE efforts, it importantly questioned the sustainability of current HIE systems.

This issue appears to be of particular concern given the low participation rates among community primary care providers.
Fontaine et al. [2] recently conducted a qualitative study of multiple small practices in Minnesota, a region with a much higher rate of EHR implementation than the national average. They found that no practice was fully involved with regional HIE; the most important barriers included cost, lack of standardized language or measures, concerns over the security of transferred information, and limited support for maintenance. Physicians also cited a lack of shared vision and sustainable business models as important barriers to HIE engagement, and many admitted to piecemeal engagement in information exchange with local stakeholders with whom they were not competing. Ultimately, the study highlighted the need for economic and leadership incentives to increase the participation of small practices in regional HIE initiatives.

These findings suggest that effective regional HIE will occur only with sustainable economic models and institutional buy-in across various types of care providers. However, there are several important challenges to these efforts. First, many existing RHIOs do not meet meaningful use standards and could have difficulties realizing the cost savings and benefits associated with HIEs. Because government funding has already been designated for further EHR development, existing RHIOs must make considerable progress to keep stride with current implementation plans and to appropriately obtain and use future funding. Given the current variability in the state of US RHIOs, more rigorous, objective evaluation of existing initiatives is needed to substantiate the efficiency, safety, and quality gains that RHIOs are believed to produce [6].

Second, sustaining financial viability beyond federal support may be a considerable challenge. Although the government has invested considerable funding in promoting the meaningful use of HIEs [7], it is clear that the funding will be temporary and that, eventually, financial responsibility will shift considerably from the federal government toward states and healthcare providers. And although the majority of RHIO participants are large hospitals rather than ambulatory practices, it is likely that buy-in from community practices will be crucial to preserving HIE funding. This buy-in will be difficult to obtain; data suggest that these smaller practices face some of the largest barriers to HIE and may be least able to secure government reimbursements via meaningful use guidelines. To address these cost and sustainability

*Corresponding author: Danny Chu, MD, FACS, Texas Heart Institute/Baylor College of Medicine, One Baylor Plaza, Houston, TX 77030, USA, Tel: 1713794 7892; Fax: 1713 794-7352; E-mail: dchumd@gmail.com

Received May 02, 2012; Accepted May 03, 2012; Published May 07, 2012

Citation: Liao JM, Chu D (2012) The State of Health Information Exchange. J Health Med Inform 3:e102. doi:10.4172/2157-7420.1000e102

Copyright: ( 2012 Liao JM, et al. This is an open-access article distributed under the terms of the Creative Commons Attribution License, which permits unrestricted use, distribution, and reproduction in any medium, provided the original author and source are credited. 
problems, some authors have suggested the creation of policies through state and RHIO partnerships, but given the short-term nature of federal funding and uncertainty regarding states' willingness to accept financial liability, there are valid concerns about how quickly, if at all, these proposed benefits can be realized.

As David Blumenthal, the former US National Coordinator for Health Information Technology, noted recently, there are other important, high-level barriers to the wider implementation and meaningful use of HIEs, including interoperability, usability, and security of health information [7]. These will all need careful attention. But given the current state of HIE and the upcoming plans to expand on existing efforts, it seems most prudent at this time to focus energy and resources on increasing the number of groups that meet national standards and piloting economic models that can leverage buy-in from a broader range of practices. Only then can the United States take the next step toward creating what everyone hopes will become a streamlined, secure, and integrated health information system.

\section{Acknowledgment}

Stephen N. Palmer, PhD, ELS, contributed to the editing of the manuscript.

\section{References}

1. Blumenthal D, Tavenner M (2010) The "meaningful use" regulation for electronic health records. N Engl J Med 363: 501-504.

2. Fontaine P, Zink T, Boyle RG, Kralewski J (2010) Health information exchange: participation by Minnesota primary care practices. Arch Intern Med 170: 622629

3. Walker J, Pan E, Johnston D, Adler-Milstein J, Bates DW, et al. (2005) The value of health care information exchange and interoperability. Health Aff (Millwood).

4. Adler-Milstein J, Bates DW, Jha AK (2011) A survey of health information exchange organizations in the United States: implications for meaningful use. Ann Intern Med 154: 666-671.

5. Tripathi M, Delano D, Lund B, Rudolph L (2009) Engaging patients for health information exchange. Health Aff (Millwood) 28: 435-443.

6. Fontaine P, Ross SE, Zink T, Schilling LM (2010) Systematic review of health information exchange in primary care practices. J Am Board Fam Med 23: 655670

7. Blumenthal D (2011) Implementation of the federal health information technology initiative. N Engl J Med 365: 2426-2431. 